\title{
Distance Preserving Flattening of Surface Sections
}

\author{
Laurent Saroul, Oscar Figueiredo, and Roger D. Hersch, Member, IEEE
}

\begin{abstract}
Curved cross-sections extracted from medical volume images are useful for analyzing nonplanar anatomic structures such as the aorta arch or the pelvis. For visualization and for performing distance measurements, extracted surface sections need to be adequately flattened. We present two different distance preserving surface flattening methods which preserve distances according to a user-specified center of interest and according to user-specified orientations. The first method flattens surface sections by preserving distances along surface curves located within planes having a user specified constant orientation. The second method flattens surfaces along curves located within radial planes crossing the center of interest. We study and compare the properties of the two flattening methods by analyzing their distortion maps. Thanks to a multiresolution approach, we provide surface flattening at interactive rates, allowing users to displace their focus point while visualizing the resulting flattened surface. These distance preserving flattening methods provide new means of inspecting curved cross-sections extracted from medical images.
\end{abstract}

Index Terms-Visualization, anatomic structures, curved sections, surface extraction, distance preserving surface flattening, interactive multiresolution flattening.

\section{INTRODUCTION}

$\mathrm{T}_{\mathrm{N}}$ the context of medical imaging, medical specialists ${ }_{\text {often need to inspect anatomic structures having a curved }}$ geometry, such as the pelvis or the aorta. Existing tools allow them to extract planar slices of any orientation traversing a structure of interest from a volume image. However, a planar slice may, for instance, not include a complete longitudinal section of a tubular curved structure such as the aorta. The extraction of curved cross-sections [10], [16] offers a new way of visualizing and inspecting curved anatomic structures. In addition, curved surfaces may easily follow structures made up of several branches such as the aorta with its three outgoing arteries (Fig. 2c) or the vena cava crossing the atrium cavity (Fig. 2d).

Textured curved surfaces may be visualized by their projections onto the viewing plane. However, such projections show some surface parts and may hide other surface parts. Surface flattening offers an alternative way of visualizing a surface section [5], [10] by enabling the visualization of all surface parts within a single planar image. However, in the general case, surface flattening introduces metric and angular distortions. In medical imaging applications, an important objective is the ability to carry out measurements for detecting anatomic abnormalities. Therefore, the flattening algorithms should be conceived so as to provide a global view of the surface and, at the same time, enable distance measurements on the flattened surface.

- L. Saroul and R.D. Hersch are with the Ecole Polytechnique Fédérale de Lausanne, School of Computer and Communication Sciences, 1015 Lausanne, Switzerland. E-mail: \{laurent.saroul, rd.hersch\}@epfl.ch.

- O. Figueiredo is with CPE Lyon, Domaine Scientifique de la Doua, Bat 308, 43 Bd du 11 Novembre 1918, BP 207769616 Villeurbanne Cedex, France.E-mail: oscar@cpe.fr.

Manuscript received 23 Dec. 2004; revised 11 Mar. 2005; accepted 19 Mar. 2005; published online 9 Nov. 2005.

For information on obtaining reprints of this article, please send e-mail to: tvcg@computer.org, and reference IEEECS Log Number TVCG-0262-1204.
In this paper, we present two different distance preserving surface flattening methods which preserve distances according to user-specified orientations. They have similar properties as known cartographic projections (Section 6). The first flattening method (Section 4) preserves distances along curves located at the intersection between the surface and planes of constant orientation specified by the user. The second algorithm (Section 5) preserves distances along curves located within radial planes crossing a center of interest (focus point). These flattening algorithms also minimize the metric and angular distortions in the proximity of the focus point.

We discuss the respective advantages and drawbacks of the two surface flattening methods by comparing their distortion maps (Section 6). We also introduce a multiresolution flattening method (Section 7) enabling surfaces to be instantly flattened. Users may therefore interactively move the center of interest within the surface section and observe the resulting changes in the flattened image. Finally, we show that flattened surface sections may be used to carry out measurements for medical purposes (Section 8).

\section{Previous Research}

Surface flattening enables professionals to visualize and inspect surfaces extracted from medical volume images. A simple but widely used technique is curved planar reformation, i.e., the extraction and flattening of a developable ruled surface [7], [3], [10]. Several ruled surfaces may be extracted and put in relation to one another [9], [10], for example, for visualizing a section of a complete vascular tree within a single planar image. These techniques have the drawback of introducing discontinuities at the junction of two different ruled surfaces. Moreover, they do not allow following highly curved and large structures such as the pelvis.

In the context of magnetic resonance images, Haker et al. [5] propose a technique for flattening the brain surface. 
They create a conformal mapping between the surface of interest and a disc. The method has also been applied to the $3 \mathrm{D}$ visualization of colon CT images [6]. However, conformal mappings, while preserving angles, create large unpredictable metric distortions.

Mlejnek et al. [12] present a flattening method for the interactive thickness visualization of articular cartilage. The method iteratively flattens triangles starting from a focus point while trying to minimize area distortions.

Other researchers addressed the problem of surface flattening in the context of texture mapping [2], [17], [18] or in the more general context of parameterization of 3D meshes [4]. However, most of these methods introduce surface cuts. For anatomic surface visualization, surface cuts are problematic since continuous surface sections are needed in order to provide an understanding of the relationships between the different anatomic structure elements. In addition, these texture mapping or surface parameterization methods do not try to preserve distances on the flattened surface.

In cartography, flattening of the sphere is the central problem. Many different cartographic projections exist [13] which each have their specific properties. For instance, the Mercator projection is conformal, the orthographic projection preserves distances on the parallels of latitude, and the Sanson projection preserves distances on the parallels of latitude and on the central meridian.

The surface flattening methods presented here have similar goals to the methods for cartographic projections. They try to preserve distances along certain orientations and minimize distortions around a point or a curve of interest. They can therefore be seen as an extension of cartographic projections from the sphere to more general curved surfaces.

In the present contribution, we extend the parallel planes surface flattening method presented in [16] by providing a multiresolution approach allowing users to interactively modify the center of a region of interest. We also present a new distance-preserving radial planes flattening method where metric distortions (Section 6) grow as a function of the distance from the center of the region of interest.

\section{Curved Surface Construction}

Let us describe the construction of a Coons surface used for extracting curved cross-sections. Given $n$ boundary cubic splines specified by the user, varying along the $u$ parameter, two other boundary cubic splines are constructed which pass through the extremities of these $n$ curves (Fig. 1, red curves). The resulting system of boundary curves is interpolated by Coons patches [8, pp. 371-382]. Starting with the $n$ curves specified by the user, we can construct $n-1$ Coons patches by carrying out, for each patch, the following interpolation:

$$
\begin{aligned}
P(u, v)= & (P(u, 0), P(u, 1))\left(\begin{array}{l}
f_{0}(v) \\
f_{1}(v)
\end{array}\right) \\
& +(P(0, v), P(1, v))\left(\begin{array}{l}
f_{0}(u) \\
f_{1}(u)
\end{array}\right) \\
& -\left(f_{0}(u), f_{1}(u)\right)\left(\begin{array}{c}
P(0,0) P(0,1) \\
P(1,0) P(1,1)
\end{array}\right)\left(\begin{array}{l}
f_{0}(v) \\
f_{1}(v)
\end{array}\right),
\end{aligned}
$$

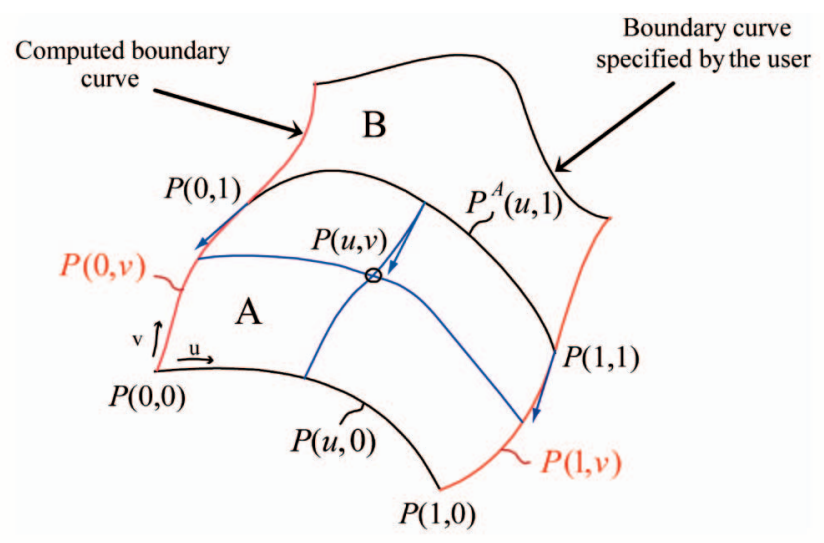

Fig. 1. Construction of a curved surface.

where $P(u, 0), P(u, 1), P(0, v)$, and $P(1, v)$ are the parametric representations of the boundary curves and where $f_{i}$ are blending functions. By choosing cubic Hermite polynomials [8, p. 379] as blending functions, the resulting patch set yields a $C^{1}$ continuous parametric surface.

Fig. 2 presents examples of curved surfaces traversing anatomic structures, projected onto the view plane. They were created thanks to an interactive applet allowing users to accurately specify surface patch boundary curves across the 3D volume image of the Visible Human [16]. Fig. 2a shows a surface passing through the left hand. Fig. $2 b$ shows a surface passing through the sternum, the costal cartilages, and the ribs. Fig. 2c shows a surface passing through the aorta, the subclavian, the carotid, and the brachiocephalic arteries. These arteries are not coplanar. Fig. $2 \mathrm{~d}$ shows a surface passing through the vena cava superior, the atrium cavity, the vena cava inferior, and several noncoplanar outgoing veins.

\section{Parallel Planes Flattening}

Parallel planes flattening preserves distances along trajectories located at the intersection between planes of a specified orientation and the surface [16]. Thanks to parallel planes flattening, medical specialists can easily measure a distance within a structure or between two structures along a trajectory located within a plane of constant orientation.

First, the user selects a point $P_{0}=P\left(u_{0}, v_{0}\right)$ on the surface $S$ as the center of his region of interest. Then, a plane orientation $H$ is chosen by the user according to the desired orientation along which distances should be preserved. The system then chooses the parametric curve $C_{u_{0}}\left\{u=u_{0}, 0 \leq v \leq v_{\max }\right\}$ (or $C_{v_{0}}$ depending on the plane orientation) $)^{1}$ on surface $S$ as the reference curve along which angular distortions are to be minimized (Fig. 3a).

By discretizing the surface for equally spaced values of $u$ (separated by a constant step size $\Delta u$, see Section 7), we obtain a set of curves $C_{u}$ with $u=$ const. For each sample point $M_{j}$ of the initial curve $C_{u_{0}}$, the plane $H_{j}$ of orientation

1. To avoid degenerate cases where a part of an isoparametric curve is parallel to the plane, we choose the set of isoparametric curves whose main orientation makes the smallest angle with the plane's normal vector. 


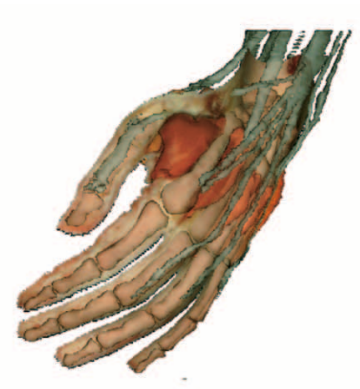

(a)

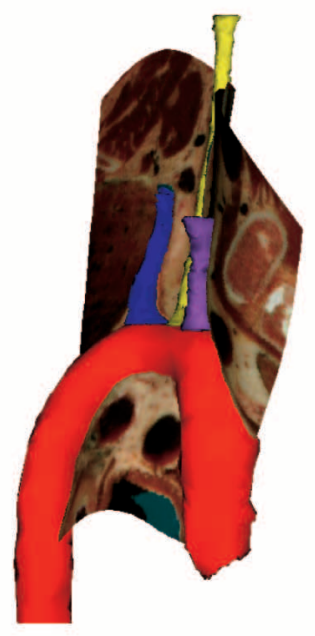

(c)

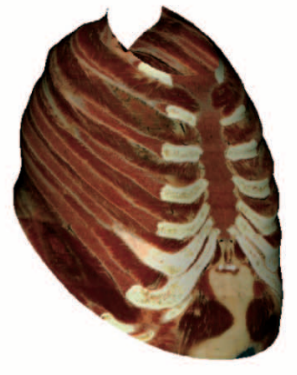

(b)

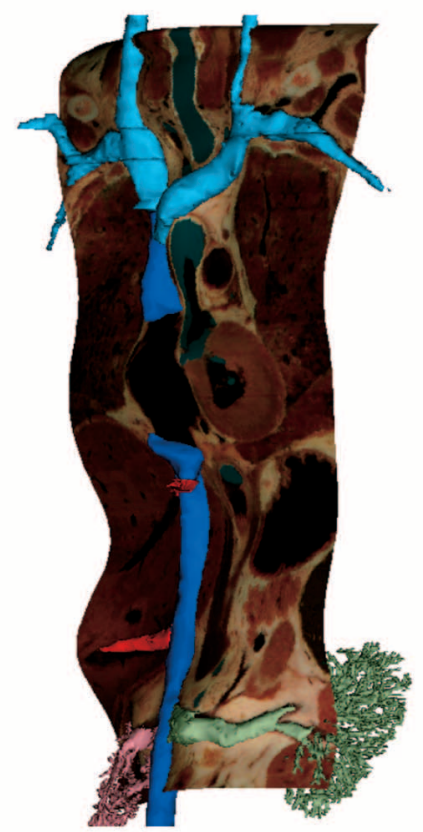

(d)
Fig. 2. Display of curved surfaces passing through anatomic structures. (a) Left hand. (b) Sternum and costal cartilages. (c) Aorta tree. (d) Vena cava tree.

$H$ passing through $M_{j}$ is computed. The intersection points between $H_{j}$ and the family of curves $C_{u}$ (Fig. 3a) provide a discrete representation of the intersection of the surface $S$ and the plane $H_{j}$ [8, pp. 507-508]. The intersection between the plane and the family of curves $C_{u}$ is computed iteratively. In the case of double intersection between the plane and a curve $C_{u}$, the system chooses the intersection point closest to the previously computed intersection point. By iterating over all sample points $M_{j}$ of $C_{u_{0}}$, we obtain a family of discrete curves $C_{j}$. Each discrete curve $C_{j}$ is located at the intersection of plane $H_{j}$ and the family of curves $C_{u}$.

With the new parameterization defined by the family of curves $C_{u}$ and the family of curves $C_{j}$, the flattening algorithm is comprised of the following steps:

1. Map the initial curve $C_{u_{0}}$ onto a plane by preserving the geodesic curvature [8, pp. 46-47] $]^{2}$ at each sample

2. The geodesic curvature $k_{g}$ of a curve $C(s)$ belonging to a surface $S$ at a point $X$ is the norm of the projection of its curvature vector at $X$ onto the tangent plane.

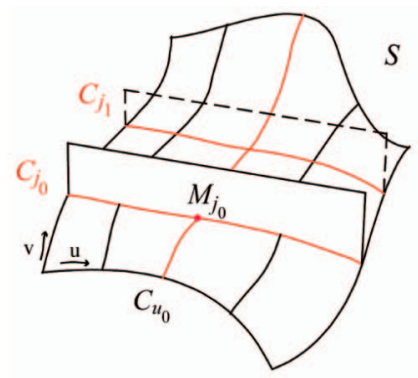

(a)

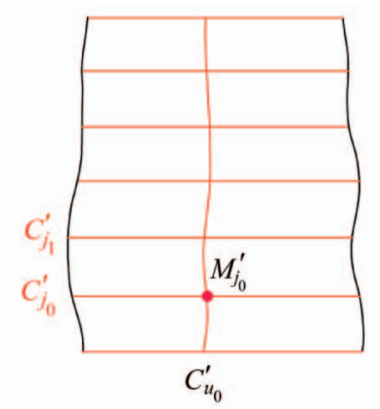

(b)
Fig. 3. Parallel flattening of a curved surface. (a) Original 3D surface. (b) Flattened surface.

point and by preserving the distance between points, ${ }^{3}$ according to [2].

2. Map a curve $C_{j_{0}}$ into a straight line $C_{j_{0}}^{\prime}$ with cross angle preservation between $C_{j_{0}}$ and $C_{u_{0}}$ and distance preservation between consecutive sample points of $C_{j_{0}}$.

3. Map each curve $C_{j}$ into a straight line parallel to $C_{j_{0}}^{\prime}$ and passing through $M_{j}^{\prime}$ with distance preservation between consecutive sample points of $C_{j}$.

By construction, this method preserves both the distances on the reference curve $C_{u_{0}}^{\prime}$ and on the transversal lines $C_{j}^{\prime}$ (Fig. 3b). The cross angle between $C_{u_{0}}$ and $C_{j_{0}}$ is also preserved. The geodesic curvature is preserved along the reference curve $C_{u_{0}}^{\prime}$. Therefore, metric distortions are minimized along a band of interest near the curve $C_{u_{0}}^{\prime}$ and both angular and metric distortions are minimized in the proximity of the focus point.

Each facet of the resulting flattened surface is sampled according to the display grid. The corresponding color texture is then extracted from the 3D volume image by nearest neighbor or trilinear interpolation.

Fig. 7 presents the flattened surface passing through the aorta arch and three outgoing arteries for two different points of reference. This flattened surface shows the connections between the aorta and the three outgoing arteries within a single planar image. Users may interactively drag the reference point to new positions (Section 7). They may also rotate the distance preservation orientation by rotating it within the flattened view. Measurements along the orientation represented by the blue straight lines (Fig. 7) may then be directly carried out on the flattened image.

\section{Radial Planes Flattening}

When analyzing anatomic structures, one may need to measure the distance between organ extremities and a point of reference. This may help, for instance, in detecting possible abnormalities. Therefore, we propose a flattening algorithm which preserves distances along trajectories located within all radial planes around a point of reference and which minimizes angular and metric distortions in the

3. Preserving the geodesic curvature along a curve $C(s)$ consists of creating a planar curve $C^{\prime}(s)$ having a curvature equal to the geodesic curvature of the original curve at each sample point. 


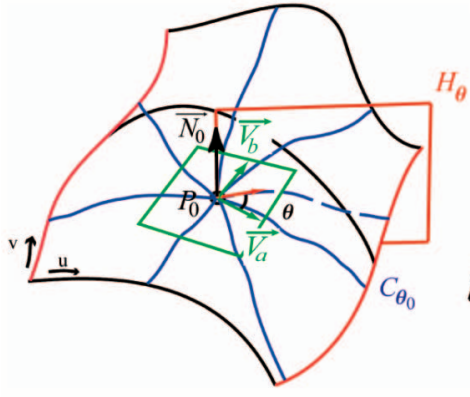

(a)

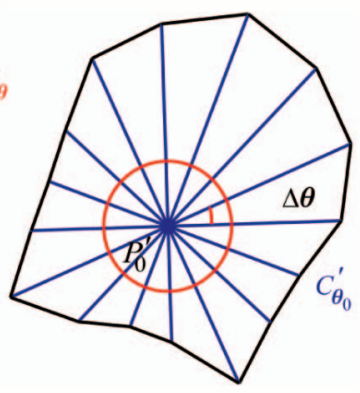

(b)
Fig. 4. The polar coordinate system on the original 3D surface and the flattened polar map. (a) Original 3D surface. (b) Flattened surface.

proximity of the focus point. We define a polar coordinate system on the curved surface centered at the point of reference $P_{0}$. Given the surface's tangential plane at the reference point and a perpendicular plane through the reference point, we construct a trajectory on the curved surface located at the intersection with this perpendicular plane. This trajectory is mapped into a straight line on the flattened surface by preserving its length.

With the surface $S$ defined by its parametric equation $P(u, v)$ and a point of reference $P_{0}=P\left(u_{0}, v_{0}\right)$ on the surface, we first compute the normal vector at $P_{0}$, $\overrightarrow{N_{0}}=\overrightarrow{P_{u}}\left(u_{0}, v_{0}\right) \times \overrightarrow{P_{v}}\left(u_{0}, v_{0}\right)$. We choose a reference vector $\overrightarrow{V_{a}}$ on the tangential plane and compute $\overrightarrow{V_{b}}=\overrightarrow{N_{0}} \times \overrightarrow{V_{a}}$. We establish a local coordinate system given by vectors $\overrightarrow{V_{a}}, \overrightarrow{V_{b}}$ and the reference point $P_{0}$. For each $\overrightarrow{V_{\theta}}=\overrightarrow{V_{a}} \cos \theta+\overrightarrow{V_{b}} \sin \theta$, we denote $H_{\theta}$, the plane perpendicular to the tangential plane, spanned by $\vec{V}_{\theta}$ and $\overrightarrow{N_{0}}$. The intersection between the plane $H_{\theta}$ and the surface $S$ is computed. In the same way as for parallel planes flattening, the resulting discrete curve is computed by intersecting the plane with a series of isoparametric curves $C_{u_{i}}\left\{u=u_{i}, 0 \leq v \leq v_{\max }\right\}$ or, respectively, $C_{v_{i}}\{0 \leq$ $\left.u \leq u_{\max }, v=v_{i}\right\}^{1}$ separated by a constant step size $\Delta u$ or, respectively, $\Delta v$ [8, pp. 507-508]. By iterating along each angular orientation $\theta_{i}$ separated by a constant angular step $\Delta \theta$, we obtain a family of discrete curves $C_{\theta_{i}}$ (Fig. 4a). Each point of the surface may then be represented by the polar coordinates $P(r, \theta)$, where $r$ is the length of the portion of the curve $C_{\theta_{i}}$ between $P_{0}$ and $P$.

With the polar system defined by the family of curves $C_{\theta_{i}}$, the radial planes flattening algorithm is comprised the following steps:

1. The point of reference $P_{0}$ is mapped onto a point $P_{0}^{\prime}=\left(x_{0}, y_{0}\right)$ on the plane.

2. An initial curve $C_{\theta_{0}}$ is mapped into a straight line by preserving the distance between sampled points.

3. Each curve $C_{\theta_{i}}$ is mapped into a straight line by preserving the distance between sampled points and by preserving the angle $\Delta \theta$ between each consecutive curve $C_{\theta_{i}}$.
We take an angle step $\Delta \theta$ that is sufficiently small (half a degree) to ensure that the surface may be linearly interpolated between two consecutive curves $C_{\theta_{i}}$ and $C_{\theta_{i+1}}$.

As in the case of parallel planes flattening, each facet of the resulting flattened surface is sampled according to the display grid and the corresponding color texture is extracted from the 3D volume image.

This resulting radial planes polar map is different from a geodesic polar map [14], [19], where distances are preserved along geodesic curves originating at the focus point. The geodesic polar map has the limitation of being applicable only within a small neighborhood of a given point [11, pp. 165-168] due to possible mutual intersections of geodesics.

Fig. 8 shows the surface passing through the aorta tree flattened according to two different reference points. Radial planes flattening minimizes the distortions around the focus point. It also allows users to directly carry out measurements along the orientations of the radial lines on the flattened image (blue lines, Fig. 8).

\section{Evaluation of the Flattening Methods by Distortion MEASUREMENTS}

In order to evaluate the advantages and drawbacks of the two flattening methods and to provide feedback about distortion magnitude and orientation, let us introduce metrics of distortion.

We rely on the distortion metrics described by Sander et al. [15] and Sorkine et al. [17]. Given a triangle of the discretized surface, the distortion caused to this triangle is measured by the singular values of the Jacobian of the affine transformation $\mathrm{S}$ between the original triangle $T$ and the corresponding mapped triangle $T^{\prime}$ located on the flattened surface. The singular values $\gamma_{\min }$ and $\gamma_{\max }$ of the Jacobian matrix $\mathbf{J}=\left[S_{x} S_{y}\right]$ are the eigenvalues of the matrix $\mathbf{J} \times \mathbf{J}^{\mathrm{T}}$. The singular values correspond to the largest and smallest scaling factors. Sander et al. [15] take the root-mean-square of the two values as the $L^{2}$ metric and $\gamma_{\max }$ as the $L^{\infty}$ metric. Sorkine et al. [17] define the distortion factor

$$
D\left(T, T^{\prime}\right)=\max \left(\gamma_{\max }, \frac{1}{\gamma_{\min }}\right)
$$

since stretching and shrinking may be considered the same for the purpose of measuring geometric distortions. If the distortion factor $D\left(T, T^{\prime}\right)$ is one, the triangles are isometric and there are no distortions. We adopt this distortion factor for our distortion measurements. We also calculate its mean value over the whole flattened surface. In addition to the distortion factor, we calculate the eigenvectors $\vec{V}_{\min }, \vec{V}_{\max }$ of the matrix $\mathbf{J} \times \mathbf{J}^{\mathrm{T}}$ corresponding to the singular values $\gamma_{\min }, \gamma_{\max }$. These vectors define the orientations of the smallest and largest scaling, i.e., the main orientations of distortions. In order to visualize distortions, we also compute and display (Figs. 7 and 8 , red lines) vectors $\vec{V}$ corresponding to the main distortion orientations, i.e., 


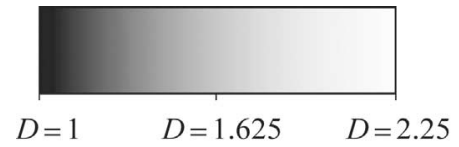

Fig. 5. Grayscale distortion factors.

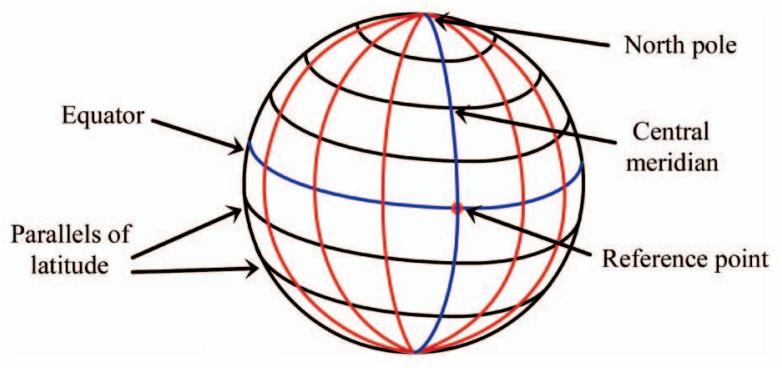

(a)

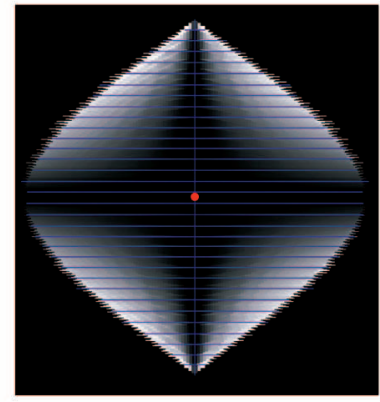

Mean distortion factor $=1.18$

(b)

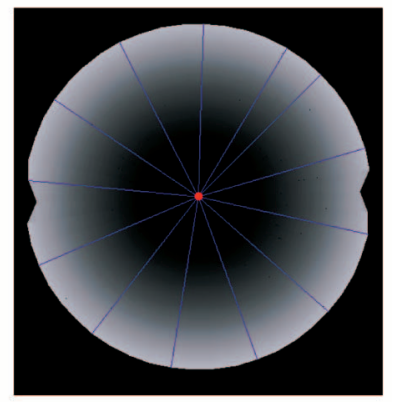

Mean distortion factor $=1.24$

(c)
Fig. 6. (b) Parallel planes and (c) radial planes flattening of (a) a hemisphere.

$$
\vec{V}= \begin{cases}\vec{V}_{\min } & \text { if } \gamma_{\max }<\frac{1}{\gamma_{\min }} \\ \vec{V}_{\max } & \text { if } \gamma_{\max } \geq \frac{1}{\gamma_{\min }}\end{cases}
$$

To illustrate the distortions induced by the two flattening methods, we apply them to a sphere (Fig. 6a). For the parallel planes flattening method (Fig. 6b), the central meridian of the sphere is taken as the curve of reference and the intersection of the central meridian and the equator as the reference point. The planes passing through the parallels of latitude of the sphere define the orientation of distance preservation. For radial planes flattening (Fig. 6c), we take the north pole as the reference point. For both flattening methods, only one half of the sphere is flattened. Fig. 6 presents the distortions maps for the two techniques with grayscale (Fig. 5) representing distortion factors.

With the parallel planes flattening method, distances are preserved along each parallel of latitude of the half sphere as well as on the central meridian. The parallels of latitude (Fig. 6a) and the central meridian (Fig. 6a) become straight lines (blue horizontal lines and the blue vertical line, Fig. 6b). Distortions increase with increasing distances from the central meridian and from the equator. These properties are exactly those of the Sanson cartographic projection [11, pp. 211212], [13]. For the presented reference curve and parallel planes layout, the Sanson projection and the parallel planes flattening method are equivalent.
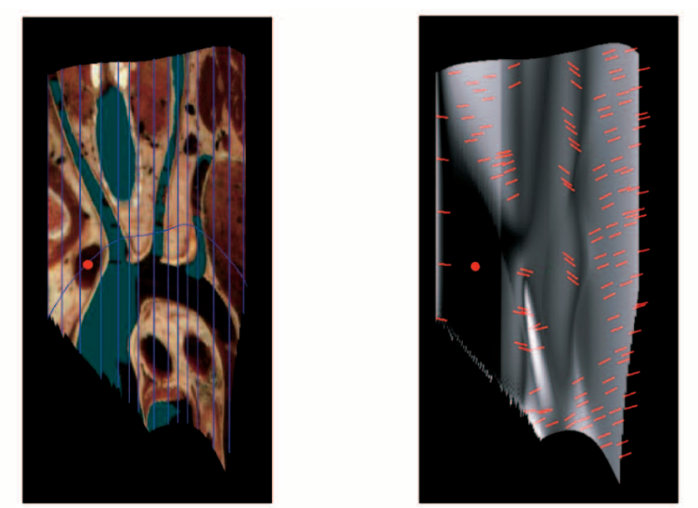

Mean distortion factor $=1.260$, maximal distortion factor $=3.5$
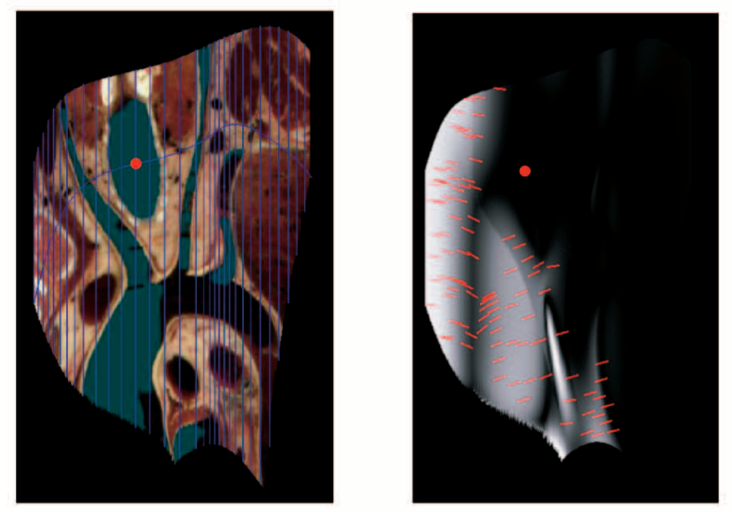

Mean distortion factor $=1.128$, maximal distortion factor $=4.04$

Fig. 7. Distortion map for parallel planes flattening.

With the radial planes flattening method, distances are preserved along each meridian of the sphere. The images of meridians (Fig. 6a, red lines) are straight lines (Fig. 6c, blue lines) while the images of the sphere's parallels of latitude are circles. The distances along parallels of latitude are preserved near the reference point and are stretched proportionally to the distance from the reference point. These properties are exactly those of the azimuthal equidistant projection used in cartography [13]. With respect to the hemisphere, the azimuthal equidistant projection and the radial planes flattening method are therefore equivalent.

Let us analyze the proposed distance preserving flattening methods on a real curved surface. Fig. 7 shows that, for parallel planes flattening, the main orientation of distortions (red segments) is orthogonal to the direction of distance preservation. The distortions are minimally close to the reference point. With the radial planes flattening method (Fig. 8), the main orientation (red segments) of distortions is also orthogonal to the lines of distance preservation (orthoradial). Near the reference point, the resulting deformations are negligible. They increase with increasing distance from the reference point.

With both flattening methods, the distortions are minimally close to the reference point. However, with parallel planes flattening, distances are preserved along one orientation and both distance and geodesic curvature are preserved along the reference curve. Distortions are therefore small in the proximity of the reference curve.

Since the distortions increase continuously with the radial planes method, in case of a very large surface such as 

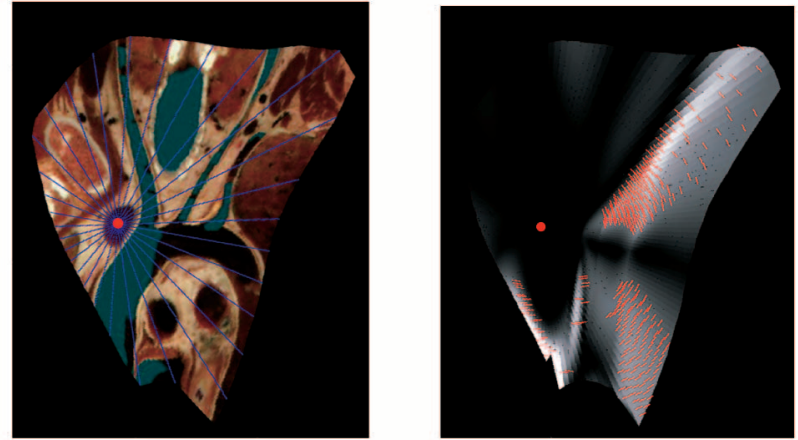

Mean distortion factor $=1.121$, maximal distortion factor $=3.6$
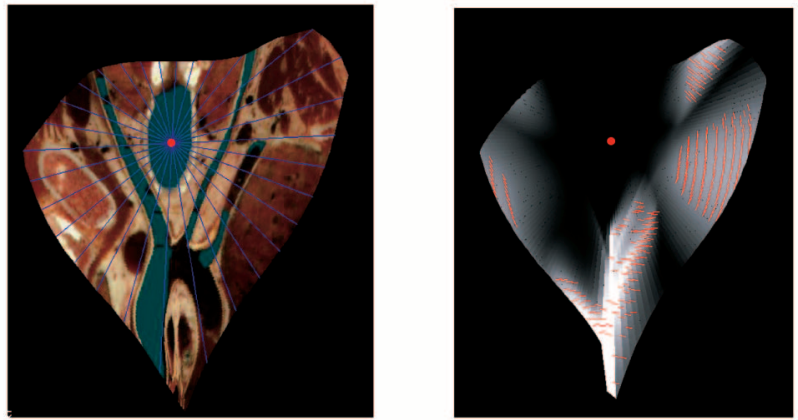

Mean distortion factor $=1.174$, maximal distortion factor $=4.39$

Fig. 8. Distortion map for radial planes flattening.

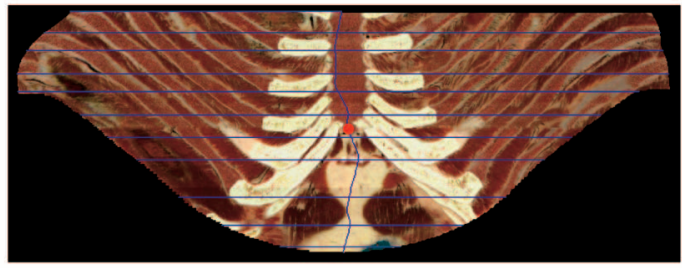

(a)

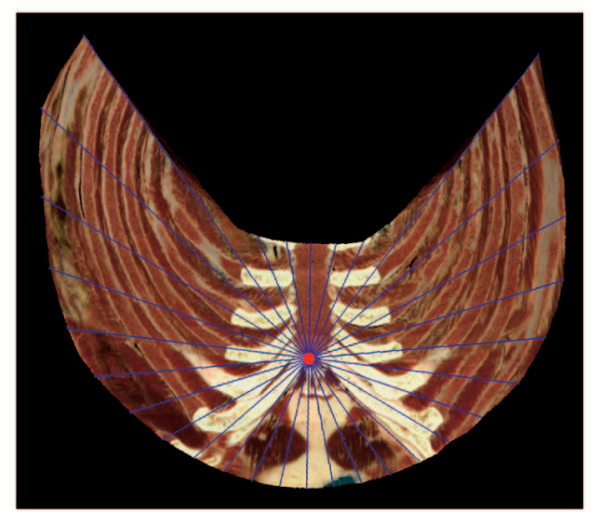

(b)

Fig. 9. Flattening of a curved surface passing through the sternum and the ribs with (a) the parallel and (b) the radial planes flattening method.

a surface passing through the sternum and ribs (Fig. 9), high distortions may occur (Fig. 9b). In the case of large surfaces, parallel planes flattening seems to be more appropriate (Fig. 9a). However, within a small neighborhood around the point of interest, radial planes flattening generally yields a locally less distorted flattened image than parallel planes flattening.

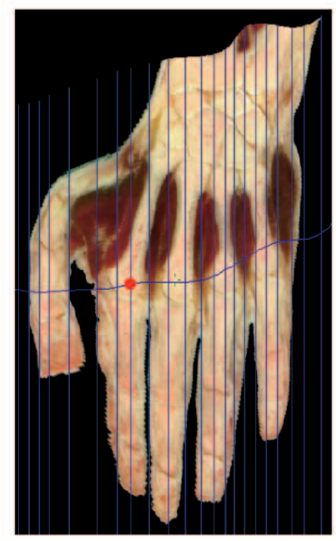

Mean distortion factor $=1.25$

(a)

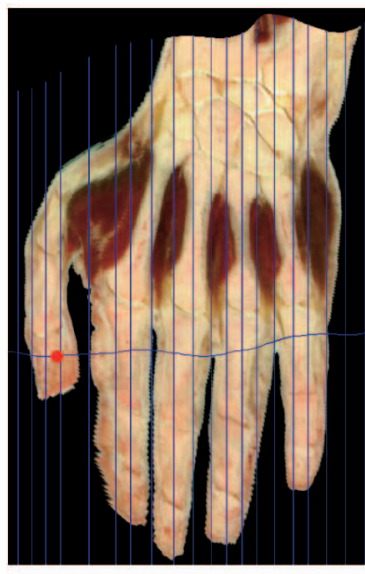

Mean distortion factor $=1.23$

(c)

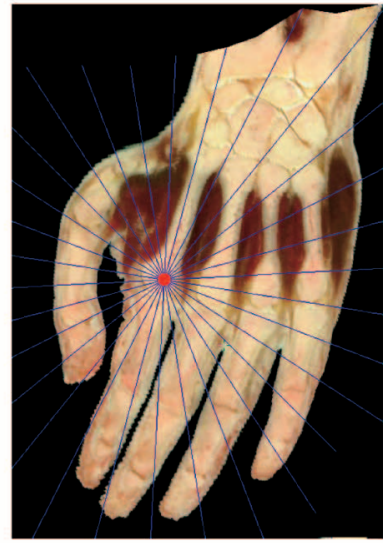

Mean distortion factor $=1.13$

(b)

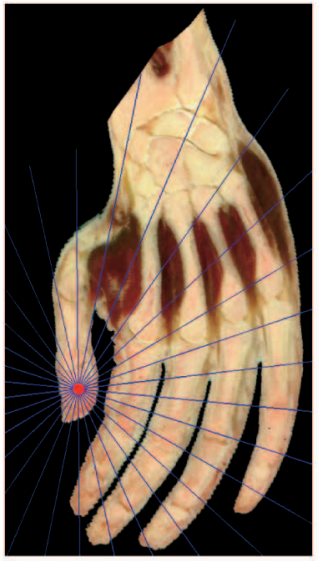

Mean distortion factor $=1.36$

(d)
Fig. 10. Flattening of the same curved surface passing through the left hand with (a) and (c) the parallel and (b) and (d) the radial planes flattening methods with two different focus points.

By construction, with the radial flattening method, a higher curvature around the point of interest yields higher distortions on other parts of the surface. Flattening of the hand is shown in Figs. 10a and 10b with the focus point located within a local flat region and, respectively, in Figs. 10c and 10d with the focus point located within a region of high curvature. Clearly, the hand flattened using the radial planes method (Fig. 10d) shows an elliptical deformation which yields higher distortions than the hand flattened using the parallel planes method (Fig. 10c). In both cases, when the focus point is located on a low curvature surface part, distortions remain small near the focus point (Figs. 10a and 10b).

As a further illustration of the two methods, Fig. 11 shows the flattened surface section passing through the vena cava tree.

\section{InTERACtive FLatTening}

We integrated the flattening algorithms into a client-server Web application (Java applet) which offers interactive tools for inspecting the anatomy of the Visible Human data set 


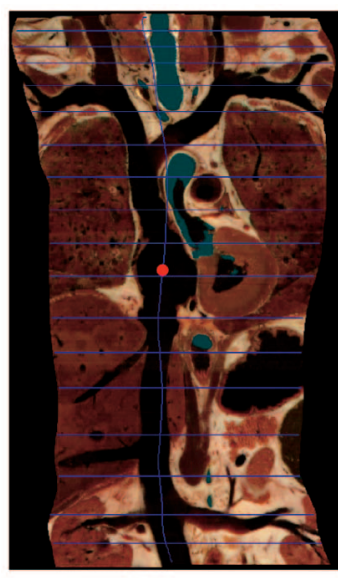

(a)

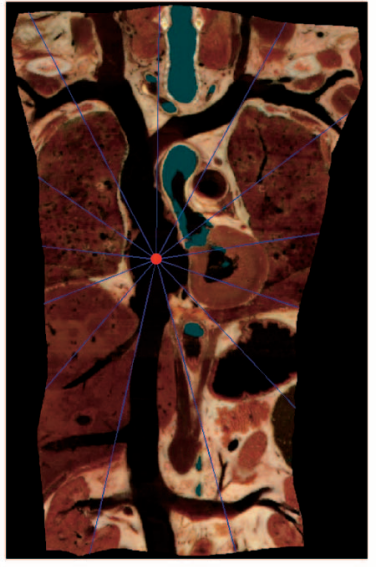

(b)
Fig. 11. Flattening of the same curved surface passing through the vena cava tree with (a) the parallel and (b) the radial planes flattening methods.

[1]. The client applet displays flattened curved surfaces extracted from the data set located on the server. Flattened surface parts are inspected by interactively moving the focus point on the flattened image. Thanks to a multiresolution approach, flattened surface images are displayed at interactive rates within the online application. We first compute the flattened surface at a coarse discretization step when the focus point is moved. When the focus point stops moving, the flattened surface description is refined by decreasing the discretization step down to the optimal discretization. We further reduce the time to produce the coarse resolution flattened surface by reusing the texture of the surface computed during the previous flattening step and by extracting the final texture from the volume data set located on the server only when the final high resolution flattened surface is to be displayed.

The considered multiresolution discretization step aims at speeding up the computation of the intersections between the surface and the family of, respectively, parallel planes $H_{j}$ and polar planes $H_{\theta}$ (Sections 4 and 5). The surface $\mathrm{S}$ is given in parametric form $P(u, v)=$ $(x(u, v), y(u, v), z(u, v))$ and the plane in implicit form $f(x, y, z)=0$. Their intersection leads to the equation

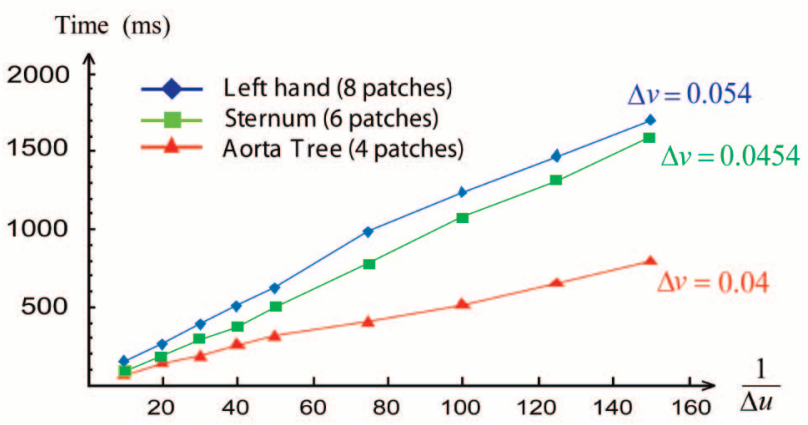

Fig. 12. Parallel planes flattening time as a function of the number of discretization steps $1 / \Delta u$ per plane intersection for parallel planes flattening.

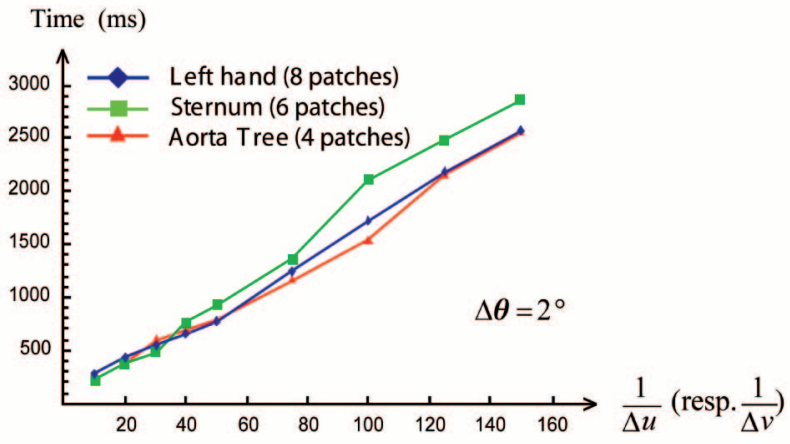

(a)

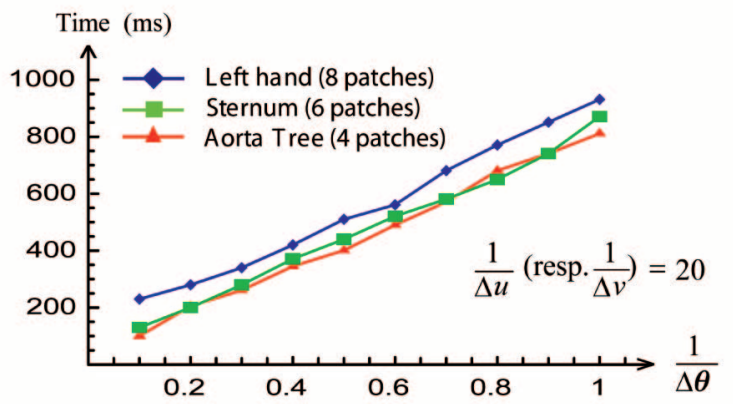

(b)

Fig. 13. Radial planes flattening time as a function of (a) the number of discretization steps per plane intersection $1 / \Delta u$ (respectively, $1 / \Delta v$ ) and (b) the number of plane intersections per degree $1 / \Delta \theta$.

$f(x(u, v), y(u, v), z(u, v))=0$. To solve this equation, we set $u=u_{i}$ (or, respectively, $v=v_{j}$ ) and find the solutions $v=v_{k}$ (respectively, $u=u_{k}$ ). We repeat this for a series of equally spaced $u$ values (respectively, $v$ values), resulting in a set of intersection points $P\left(u_{i}, v_{k}\right)$ (or, respectively, $P\left(u_{k}, v_{i}\right)$ ) [8, pp. 507-508]. This is equivalent to the computation of the intersection between the plane and a set of isoparametric curves $C_{u_{i}}\left\{u=u_{i}, 0 \leq v \leq v_{\max }\right\}$ or, respectively, $C_{v_{i}}\left\{0 \leq u \leq u_{\max }, v=v_{i}\right\}$, separated by a constant step size $\Delta u$ or, respectively, $\Delta v$ (Sections 4 and 5). If the set of curves is sufficiently dense, i.e., the step size is sufficiently small, the resulting set of intersection points $P_{u_{i}}\left(\right.$ or $P_{v_{i}}$ ) provides a piecewise linear approximation of the intersection between the plane and the surface. In order to reduce computation times during the displacement of the focus point, the multiresolution approach consists in modifying the step size providing the discrete intersection between one plane and the surface as well as the step size controlling the number of intersecting planes. For radial planes flattening, the number of planes depends on the angular discretization step size $\Delta \theta$. For parallel planes flattening, the number of planes depends on the discretization step $\Delta v$ along the reference curve $C_{u_{0}}$ (see Fig. 3).

Figs. 12 and 13 give the flattening times (P4 1.7 GHz, 512 Mo Ram) for three different surfaces, with the left hand (Fig. 10) incorporating eight patches, the aorta (Figs. 7 and 8) comprising four patches, and the sternum and costal cartiledge surfaces (Figs. 15 and 16) comprising six patches. 
TABLE 1

Examples of Step Sizes, Number of Mesh Triangles, and Computation Times for Parallel Planes Flattening

\begin{tabular}{|l|c|c|c|c|c|}
\hline Surfaces & $\begin{array}{c}\Delta u_{\text {opt }}, \Delta v_{\text {opt }} \\
(\mathrm{nb} \text { triang. })\end{array}$ & $t_{\text {opt }}$ & $\begin{array}{c}\Delta u_{\min }, \Delta v_{\min } \\
(\mathrm{nb} \text { triang. })\end{array}$ & $t_{\min }$ & $\begin{array}{c}\text { Frame } \\
\text { rate }\end{array}$ \\
\hline $\begin{array}{l}\text { Left Hand } \\
\text { (8 patches) }\end{array}$ & $\begin{array}{c}0,0119 / 0,054 \\
(20676 \mathrm{~T})\end{array}$ & $1125 \mathrm{~ms}$ & $\begin{array}{c}0,02822 / 0,128 \\
(3264 \mathrm{~T})\end{array}$ & $188 \mathrm{~ms}$ & 5,31 \\
\hline $\begin{array}{l}\text { Sternum } \\
\text { (6 patches })\end{array}$ & $\begin{array}{c}0,022 / 0,0454 \\
(11234 \mathrm{~T})\end{array}$ & $470 \mathrm{~ms}$ & $\begin{array}{c}0,03373 / 0,0696 \\
(4528 \mathrm{~T})\end{array}$ & $204 \mathrm{~ms}$ & 4,9 \\
\hline $\begin{array}{l}\text { Aorta tree } \\
\text { (4 patches) }\end{array}$ & $\begin{array}{c}0,0166 / 0,04 \\
(9958 \mathrm{~T})\end{array}$ & $375 \mathrm{~ms}$ & $\begin{array}{c}0,0228 / 0,0548 \\
(5098 \mathrm{~T})\end{array}$ & $189 \mathrm{~ms}$ & 5,29 \\
\hline
\end{tabular}

For both flattening methods, the flattening time is proportional to the number of discretization steps used for computing the discrete intersections between the plane and the surface. The radial planes flattening time is also proportional to the number of planes per unit of angle. By construction, the parallel planes flattening time is also proportional to the number of planes per unit length.

Since the Coons surface is defined by interpolating the boundary curves, we compute the optimal step size $\Delta u_{\text {opt }}$ (respectively, $\Delta v_{\text {opt }}$ ) by recursive subdivision of the step sizes of each boundary spline curve $P(u, i)$ (respectively, $P(k, v)$ ) until the height of each triangle formed by three consecutive sample points of the spline curve is smaller than the data set pixel size. The smallest step size from all surface patches becomes $\Delta u_{o p t}$ (respectively, $\Delta v_{\text {opt }}$ ). Regarding the angular step size $\Delta \theta$, experience shows that a step size $\Delta \theta_{\text {opt }}=0.5^{\circ}$ results in a high quality flattened surface.

Experience shows that, in order to provide interactivity when moving the reference point, it is necessary to display at least five flattened images per second. With the optimal step sizes $\Delta u_{o p t}$ and $\Delta v_{o p t}$ (and $\Delta \theta_{o p t}$ for radial planes flattening), we measure the time $t_{\text {opt }}$ to flatten the surface. For parallel planes flattening, we derive the step sizes $\Delta u_{\text {min }}$ and $\Delta v_{\min }$ yielding the desired interactive flattening time. Regarding radial planes flattening, experience also shows that the angular step size $\Delta \theta$ must be less than six degrees in order to ensure a sufficiently good quality for the low resolution surface discretization. We, therefore, first compute the minimal step sizes $\Delta \theta_{\min }$ and $\Delta u_{\min }$ (respectively, $\left.\Delta v_{\min }\right)$. If the upper bound of $\Delta \theta$ is reached, we take $\Delta \theta_{\min }=6^{\circ}$ and derive the step sizes $\Delta u_{\min }$ (or, respectively, $\left.\Delta v_{\min }\right)$ yielding the desired interactive computation time $t_{\max } \simeq 200 \mathrm{~ms}$. Examples of step sizes, corresponding number of mesh triangles, and measured computation times are given in Table 1.

When the center of interest reaches its final position, we flatten the surface according to the optimal discretization steps and fill the flattened image with the locally available texture. We then request the final texture from the server and generate the final high resolution flattened image.

The differences between the low resolution flattened surface and the final flattened surface are only significant at
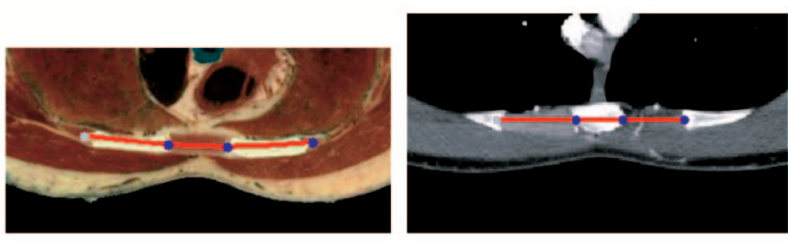

Fig. 14. Curve control points at the extremities of the costal cartilages.

a large distance from the center of interest. Therefore, during interaction, image quality remains generally high for most of the surfaces. However, in the case of large and highly curved surfaces, the computation time may be too important to ensure both interactivity and high quality.

Multiresolution surface flattening enables the system to compute several flattened images per second and, therefore, provides a progressive and continuous deformation of the flattened surface according to the displacement of the focus point.

\section{Carrying Out Measurements along FLATTENEd SURFACES}

Let us compare different anatomies by measuring distances on flattened curved surfaces laid out across the same anatomic reference points. We consider two different volume data sets. The first data set is the Visible Human cryosection data set, a 15 GB true color 3D volume [1] sampled at a resolution of $3 \times 3 \times 1$ voxels per $\mathrm{mm}^{3}$ on, respectively, the $x, y$, and $z$ axis. The second data set is a $100 \mathrm{MB}$ computer tomography volume data set (courtesy of the University Hospital of Lausanne, Dr. Reto Meuli) sampled at a resolution of $1 \times 1 \times 1 / 3$ voxels per $\mathrm{mm}^{3}$ on, respectively, the $x, y$, and $z$ axis. We extract from both data sets a similar surface defined by the same anatomic reference points and compare the two resulting flattened images.

We extract a surface from each data set passing through the sternum and the costal cartilages. Each surface is constructed by specifying curve control points at the two extremities of the intersection between axial slices and the costal cartilages (Fig. 14). For each costal cartilage pair, we choose the axial slice which passes through the external extremities of the costal cartilages.

Parallel planes flattening (Fig. 15) is carried out by preserving distances along intersections between the surface and axial planes. With both flattening techniques, the flattened surfaces obtained from the Visible Human and from the CT images are similar. With parallel planes flattening (Fig. 15), distances measured along the horizontal orientation (green lines orientations) may be compared. With radial planes flattening (Fig. 16), the distances between the reference point and another structure within a radial direction (green lines) may be compared. These measurements may help specialists in characterizing possible anatomic abnormalities provided that the corresponding anatomic structures having clearly identifiable anatomic reference points.

A curved surface defined by control points located on anatomic reference points provides a stable reference frame 

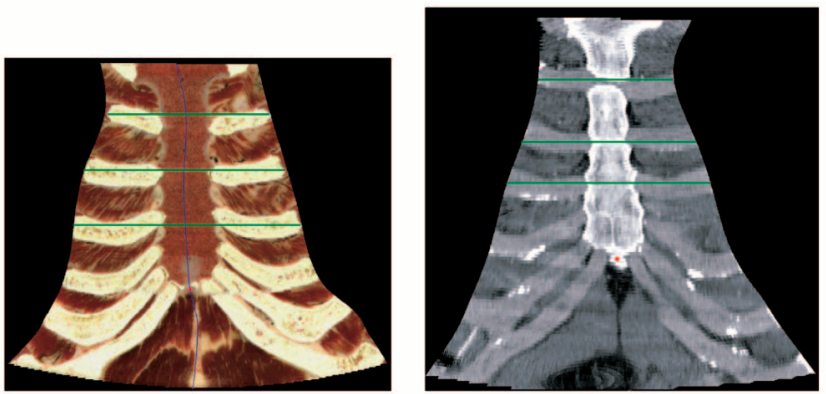

Fig. 15. Comparing the sternum and costal cartilages of the Visible Human and of a CT data set thanks to surfaces flattened according to parallel planes flattening.

for measurements. This may prove to be particularly useful for following the evolution of dynamically growing structures such as tumors.

To characterize such an evolution, besides distance measurements on the flattened surface, medical specialists may want to measure the perimeter or the area of a structure intersected by the curved surface. The flattened view may therefore provide users with a simple means of performing such measurements. Once a curve following the boundary of the structure of interest on the flattened surface has been specified, the corresponding 3D curve on the original surface may be easily tracked and its length computed. In the case of a closed curve, the corresponding enclosed surface area may also be computed.

\section{Conclusion}

We introduce two interactive surface flattening methods for visualizing curved cross-sections extracted from medical volume images. These methods enable the interactive visualization of a flattened curved surface and, therefore, provide the means for a thorough inspection of anatomic structures.

Parallel planes flattening preserves distances along the intersection between parallel planes of constant orientation and the surface. Radial planes flattening preserves distances along trajectories located at the intersection between the surface and radial planes passing through the center of a region of interest. These distance preserving flattening methods may enable specialists to establish the differences between different anatomic morphologies.

We illustrate the properties of the flattening methods by using distortions maps displaying the intensity and main orientation of distortions within the flattened surfaces. By applying the flattening methods to the hemisphere, we show that they are equivalent to well-known cartographic projections.

The two proposed flattening methods minimize geometric distortions around the center of a region of interest located on the surface. In addition, parallel planes flattening also minimizes distortions along a reference curve interpolating between surface patch boundary curve. Thanks to a multiresolution approach, we flatten the surface at interactive rates, thereby enabling the real-time displacement of the center of interest. Users may inspect the different surface parts without noticeable local distortions
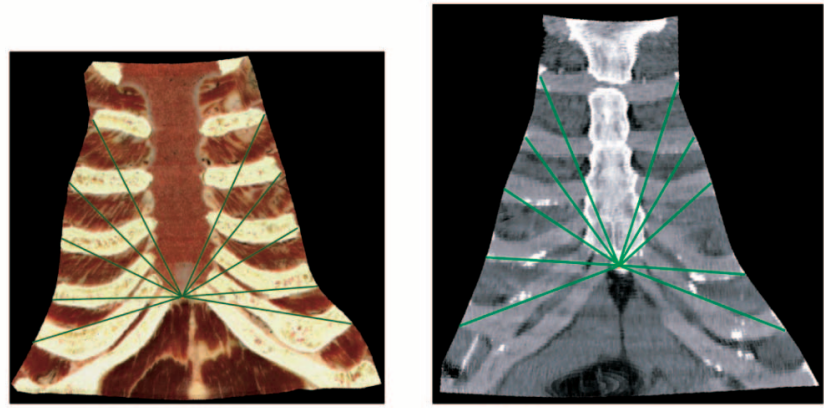

Fig. 16. Comparing the sternum and costal cartilages of the Visible Human and of a CT data set thanks to surfaces flattened according to radial planes flattening.

by displacing the reference point and observe the continuous deformation of the flattened surface.

The presented methods may provide medical specialists with new tools for visualizing and analyzing anatomic structures. They may use them for comparing morphologies or to inspect anatomic structures of patients. Distance measurements carried out on flattened surfaces may also help in detecting anatomic abnormalities. The distance preserving real time surface flattening tools are available online on our Visible Human Website (http://visiblehuman.epfl.ch). They run within a Java applet. We invite medical specialists to use them and to evaluate their possible benefits.

\section{ACKNOWLEDGMENTS}

The authors would like to thank Professor J.P. Hornung, Dr. B. Riederer, and their students from the Medical Faculty of the University of Lausanne for their advice and collaboration. They also thank Dr. Reto Meuli from the University Hospital of Lausanne (CHUV) for the tomographic data set, Dr. Peter Ratiu from Harvard Medical School for the curved surface passing through the Vena Cava tree, and Sebastian Gerlach from EPFL for his help in the development of the surface flattening Java applet.

\section{REFERENCES}

[1] M. Ackerman, "The Visible Human Project," Proc. IEEE, vol. 86, no. 3, pp. 504-511, 1998.

[2] C. Bennis, J.M. Vézien, and G. Iglésias, "Piecewise Surface Flattening for Non-Distorted Texture Mapping," Proc. Int'l Conf. Computer Graphics and Interactive Techniques, vol. 25, no. 4, pp. 237246, 1991.

[3] O. Figueiredo and R.D. Hersch, "Parallel Unfolding and Visualization of Curved Surfaces Extracted from Large Three Dimensional Volumes," J. Electronic Imaging, vol. 11, no. 4, pp. 423-433, 2002.

[4] M.S. Floater and K. Hormann, "Surface Parameterization: A Tutorial and Survey," Advances on Multiresolution in Geometric Modelling, N. Dodgson, M.S. Floater, and M. Sabin, eds., pp. 157186, Heidelberg: Springer-Verlag, 2005.

[5] S. Haker, S. Angenent, A. Tannenbaum, and R. Kikinis, "On the Laplace-Beltrami Operator and Brain Surface Flattening," IEEE Trans. Medical Imaging, vol. 18, pp. 700-711, 1999.

[6] S. Haker, S. Angenent, A. Tannenbaum, and R. Kikinis, "Non Distorting Flattening for Virtual Colonoscopy," Proc. Third Int'l Conf. Medical Robotics, Imaging and Computer Assisted Surgery, 2000.

[7] S. He, R. Dai, B. Lu, C. Cao, H. Bai, and B. Jing, "Medial Axis Reformation: A New Visualization Method for CT Angiography," Academic Radiology, vol. 8, pp. 726-733, 2001. 
[8] J. Hoschek and D. Lasser, Fundamentals of Computer Aided Geometric Design. AK Peters, 1993.

[9] A. Kanitsar, D. Fleischmann, R. Wegenkittl, P. Felkel, and M.E. Gröller, "CPR-Curved Planar Reformation," Proc. IEEE Visualization 2002 (Vis'02), pp. 37-44, 2002.

[10] A. Kanitsar, D. Fleischmann, R. Wegenkittl, P. Felkel, and M.E. Gröller, "Advanced Curved Planar Ref-ormation: Flattening of Vascular Structures," Proc. IEEE Visualization 2003 (Vis'03), pp. 4350, 2003.

[11] E. Kreysig, Differential Geometry. Dover Publications, Inc., 1991.

[12] M. Mlejnek, A. Vilanova, and M.E. Gröller, "Interactive Thickness Visualization of Articular Cartilage," Proc. Visualization 2004 (Vis'04), pp. 521-527, 2004.

[13] F. Pearson, B. Nordenskjold, and T.J. Lam, Map Projections: Theory and Applications. CRC Press, 1990.

[14] K. Polthier and M. Schmies, "Geodesic Flow on Polyhedral Surfaces," Proc. Joint Eurographics and IEEE TCVG Symp. Visualization, pp. 179-188, 1999.

[15] P. Sander, J. Snyder, S.J. Gortler, and H. Hoppe, "Texture Mapping Progressive Meshes," Proc. SIGGRAPH, pp. 409-416, 2001.

[16] L. Saroul, S. Gerlach, and R.D. Hersch, "Exploring Curved Anatomic Structures with Surface Sections," Proc. IEEE Visualization 2003 (Vis'03), pp. 27-34, 2003.

[17] O. Sorkine, D. Cohen-Or, R. Goldenthal, and D. Lischinsky, "Bounded-Distortion Piecewise Mesh Parameterization," Proc. IEEE Visualization 2002 (Vis'02), pp. 355-362, 2002.

[18] G. Zigelman, R. Kimmel, and N. Kiryati, "Texture Mapping Using Surface Flattening via Multidimensional Scaling," IEEE Trans. Visualization and Computer Graphics, vol. 8, no. 2, pp. 198-207, Apr.June 2002

[19] W. Welch and A. Witkin, "Free-Form Shape Design Using Triangulated Surfaces," Proc. SIGGRAPH, pp. 247-256, 1994.

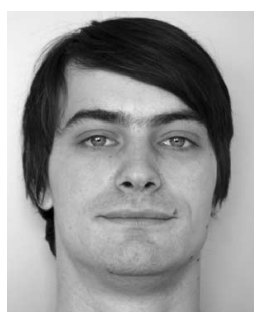

Laurent Saroul graduated in 2001 as an electronics and physics engineer from CPELyon, a French engineering school located in Lyon, France. The same year, he received the master's degree in image processing and computer graphics from the Université Jean Monnet, St-Etienne, France. Since 2002, he has worked at the Peripheral Systems Laboratory of the Ecole Polytechnique Fédérale de Lausanne, Switzerland, as a research assistant and $\mathrm{PhD}$ candidate. His current research interests include image processing, computer graphics, and volume visualization techniques.

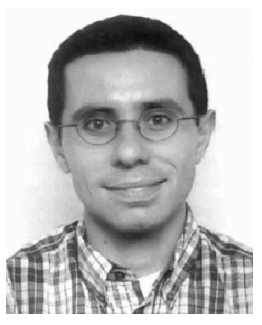

Oscar Figueiredo received the $\mathrm{PhD}$ degree in computer science in 1999 from the Ecole Polytechnique Fédérale de Lausanne (EPFL). $\mathrm{He}$ is also a CPE Lyon graduate and received the master's degree in computer graphics from the University of St-Etienne. He heads the Computer Science and Telecommunications Laboratory at the Ecole Supérieure de Chimie Physique Electronique (CPE Lyon) in France. His research interests include theoretical aspects of discrete geometry, their practical applications in computer graphics, and high-performance parallel algorithms for visualization and internet applications.

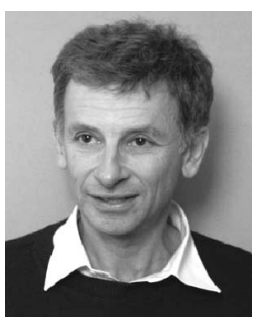

Roger D. Hersch received the engineering and PhD degrees from ETH Zurich in 1975 and from EPFL in 1985, respectively. He is a professor of computer science and head of the Peripheral Systems Laboratory at the Ecole Polytechnique Fédérale de Lausanne, Switzerland. He has published more than 100 scientific papers, is the editor of four books, and is the inventor or coinventor in many patent applications. $\mathrm{He}$ is interested in novel imaging techniques (color prediction, color reproduction, artistic imaging, security printing) and in high-performance server applications (imaging servers, PC clusters, etc.). He is a member of the IEEE and the IEEE Computer Society.

For more information on this or any other computing topic, please visit our Digital Library at www.computer.org/publications/dlib. 